\title{
Preenchimento da caderneta de saúde da criança e antropometria de crianças
}

\author{
Filling of child health handbook and anthropometric status of children
}

\author{
Llenado del cuaderno de salud del niño y antropometría de niños
}

Dixis FIGUEROA PEDRAZA ${ }^{(1)}$

${ }^{(1)}$ Universidade Estadual da Paraíba, Campina Grande, Paraíba, Brasil.

Recebido: 13 ago 2019 Revisado: 18 ago 2019 Aceito: 22 ago 2019

Autor de correspondência: Dixis Figueroa Pedrazza dixisfigueroa@gmail.com

Conflito de interesses: Os autores declaram não haver nenhum interesse profissional ou pessoal que possa gerar conflito de interesses em relação a este manuscrito.

\begin{abstract}
Resumo
Descrever o preenchimento da Caderneta de Saúde da Criança (CSC) e analisar sua relação com o estado nutricional de crianças assistidas em creches. Estudo transversal desenvolvido com 402 crianças de 24 a 72 meses de idade assistidas em creches de Campina Grande, Paraíba, Brasil. O estado nutricional foi avaliado pelos índices estatura/idade, peso/idade e IMC/idade. Verificou-se a adequação de 17 variáveis da CSC. Analisou-se a relação do escore de preenchimento da CSC com os escores-Z dos índices antropométricos.: As variáveis que apresentaram maiores frequências de preenchimento incorreto foram o registro do peso e perímetro cefálico na curva de crescimento e o registro do desenvolvimento neuropsicomotor. Crianças com preenchimento adequado da CSC tiveram maior média de estatura/idade. Os achados apresentados evidenciam deficiências de preenchimento da CSC entre crianças assistidas em creches que compreendem parâmetros do crescimento e desenvolvimento. Sugere-se o desenvolvimento de pesquisas para testar a associação entre essas deficiências com o crescimento da criança.
\end{abstract}

Descritores: Registros de Saúde Pessoal; Saúde da Criança; Promoção da Saúde; Estado Nutricional; Crescimento e Desenvolvimento.

\begin{abstract}
To describe the filling of the Child Health Handbook $(\mathrm{CHH})$ and to analyze it's relation with children nutritional status enrolled at day care centers. Cross-sectional study with 402 children 24-72 months enrolled at day care centers in the city of Campina Grande, Paraíba, Brazil. The nutritional status was assessed by height/age, weight/age and BMI/age indices. It was verified the adequacy of 17 variables of the $\mathrm{CHH}$. It was analyzed the relation between the filling score of $\mathrm{CHH}$ and the scores- $\mathrm{Z}$ of anthropometric indices. The variables with worst filling were the filling of weight and cephalic perimeter in the growth curve, as well as the filling of neuropsicomotor development. Children with adequate filling of $\mathrm{CHH}$ had higher mean of height/age. The findings show lack of CSC completion among children attending daycare centers that include growth and development parameters. We suggest the development of research to test the association between these deficiencies and the child's growth.
\end{abstract}

Keywords: Health Records, Personal; Child Health; Health Promotion; Nutritional Status; Growth and Development. 


\section{Resumen}

Describir el llenado del Cuaderno de Salud del Niño (CSN) y analizar su relación con el estado nutricional de niños asistidos en jardines infantiles. Estudio transversal desarrollado con 402 niños de 24 a 72 meses de edad que frecuentan jardines infantiles de Campina Grande, Paraíba, Brasil. El estado nutricional se evaluó usando los índices estatura/edad, peso/edad y IMC/edad. Fue verificada la adecuación de 17 variables del CSN. Se analizó la relación del escore de llenado del CSN con los escores- $Z$ de los índices antropométricos. Las variables que presentaron mayores frecuencias de llenado incorrecto fueron el registro de peso y perímetro cefálico en la curva de crecimiento y el registro de desarrollo neuropsicomotor. Niños que tuvieron CSN llenados de manera inadecuada presentaron mayor media de estatura/edad. Los resultados presentados evidencian deficiencias en el llenado del CSN entre niños asistidos en jardines infantiles que comprenden parámetros del crecimiento y desarrollo. Se sugiere el desarrollo de investigaciones para testar la asociación de esas deficiencias con el crecimiento del niño.
\end{abstract}

Palabras-claves: Registros de Salud Personal; Salud del Niño; Promoción de la Salud; Estado Nutricional; Crecimiento y Desarrollo.

\title{
Introdução
}

A utilização do Cartão da Criança - CC no Brasil data da década de 1980, quando da implantação do Programa de Assistência Integral à Saúde da Criança como proposta de unificar as ações básicas de saúde. Esse instrumento foi revisado em 2005, com modificações no seu conteúdo, quando passou a ser denominado Caderneta de Saúde da Criança - CSC e inseriu uma nova concepção na perspectiva de vigilância integral à saúde. ${ }^{1}$

Após várias reformulações, a atual CSC, diferentemente do CC, constitui um instrumento de vigilância capaz de integrar várias das ações da atenção básica à saúde da criança. $^{2}$ No contexto do estado nutricional, a CSC é considerada o melhor instrumento para o acompanhamento do crescimento da criança mediante o registro periódico do peso, da estatura e do Índice de Massa Corporal. Além disso, destaca-se por conter as curvas de crescimento desenvolvidas pela Organização Mundial da Saúde, recomendadas atualmente para o acompanhamento do crescimento. ${ }^{1,3}$

Para que a CSC cumpra seu papel na comunicação, educação, vigilância e promoção da saúde infantil, é necessário o registro correto e completo das informações pelos profissionais da saúde responsáveis pela assistência. ${ }^{2}$ A utilização da CSC de maneira adequada facilita o diálogo entre as famílias e os profissionais, possibilita a adesão e 
valorização do instrumento por parte da família e contribui na qualidade da atenção às crianças ofertadas pelos serviços de saúde. ${ }^{2,4,5}$ Nesse contexto, o monitoramento do crescimento é ação básica e pode fornecer parâmetros importantes da situação de saúde e das condições de vida da criança. ${ }^{6,7}$

Estudos sobre o preenchimento da CSC apontam dificuldades variadas e conjunturais, inclusive relativas à vigilância do crescimento, que incluem a desvalorização da caderneta pelos usuários, a falta de capacitação dos profissionais e a subutilização do instrumento por muitos dos membros da equipe de saúde. ${ }^{1,2}$ No que se refere aos registros relacionados ao crescimento, a ausência ou incompletude das informações é ressaltada. ${ }^{8}$ Entretanto, apesar de se reconhecer os benefícios da vigilância do crescimento e das creches para o estado nutricional das crianças, $^{9-11}$ os estudos sobre o preenchimento da CSC não têm delimitado o grupo de crianças nessas instituições nem possíveis associações com o estado nutricional. ${ }^{8}$ Crianças assistidas em creches apresentam particularidades relacionadas ao compartilhamento do cuidado e à inserção da mulher no mercado de trabalho que a limita no tempo dedicado aos filhos. ${ }^{12}$ Portanto, o presente estudo teve como objetivo descrever o preenchimento da CSC e analisar sua relação com o estado nutricional de crianças assistidas em creches.

\section{Metodologia}

Foi realizado um estudo transversal de abordagem quantitativa para avaliar o preenchimento da CSC de crianças de zona urbana com idade entre 24 e 72 meses matriculadas em creches públicas do município de Campina Grande, Paraíba. A coleta dos dados foi realizada nas creches entre fevereiro e março de 2013 por estudantes e profissionais da área de saúde que passaram por treinamento e participaram de estudo piloto numa das creches (106 crianças) que foi previamente selecionada de forma aleatória. 
O município de Campina Grande possui área geográfica de $641 \mathrm{~km}^{2}$ e 376.133 habitantes, sendo sede do Terceiro Núcleo Regional de Saúde, uma macrorregional estadual de saúde que congrega 70 municípios e é referência para os serviços de saúde, ainda, para os estados de Pernambuco e Rio Grande do Norte. O Programa Saúde da Família de Campina Grande foi implantado em 1994, compondo, com mais 13 municípios do país, as experiências pioneiras institucionalizadas pelo Ministério da Saúde. ${ }^{13}$ Possuía, na época da pesquisa, rede básica de saúde com 92 equipes de saúde da família distribuídas em seis distritos sanitários e cobertura de $85 \%$.

No momento da coleta dos dados, funcionavam 25 creches públicas (2.931 crianças inscritas) distribuídas em áreas carentes de bairros distintos do município. Das 25 creches, uma foi escolhida para o estudo piloto e três que estavam em reforma (fechadas e crianças não remanejadas) consideraram-se não aptas para o estudo. Além disso, as duas creches da zona rural foram excluídas, considerando as especificidades do processo de transição nutricional das crianças do meio rural. Nas 19 creches disponíveis para o estudo, foram excluídas as crianças atendidas no modelo de berçário (abaixo da idade mínima do estudo), gêmeas (risco para nascimento baixo peso e comprometimento do crescimento), adotadas (sem informações sobre a gestação e nascimento), de mães menores de 18 anos (risco para nascimento baixo peso e comprometimento do crescimento) e aquelas com problemas físicos que comprometessem a avaliação antropométrica, o que totalizou 1820 crianças elegíveis. Considerando-se prevalência estimada de preenchimento incorreto da CSC de $30 \%,{ }^{2}$ erro amostral de 5\%, nível de 95\% de confiança $\left(Z \alpha^{2}=1,96^{2}\right)$, poder da amostra de $80 \%$, perdas na ordem de $10 \%$ e efeito do desenho de 1,4 , estimou-se a necessidade de estudar 426 crianças, que foram selecionadas por sorteio aleatório simples. Os responsáveis das crianças sorteadas foram convidados a comparecer às creches portando a CSC, das quais 16 não portaram o instrumento e oito não aceitaram 
participar da pesquisa, conformando-se assim a amostra de 402 crianças do estudo, cujas CSC foram analisadas em relação ao preenchimento (Figura 1).

Figura 1. Fluxograma de seleção da população de estudo (total de crianças, excluídas, elegíveis, perdidas e estudadas) para descrever o preenchimento da Caderneta de Saúde da Criança e sua relação com o estado nutricional antropométrico de crianças. Campina Grande, Paraíba, Brasil, 2013

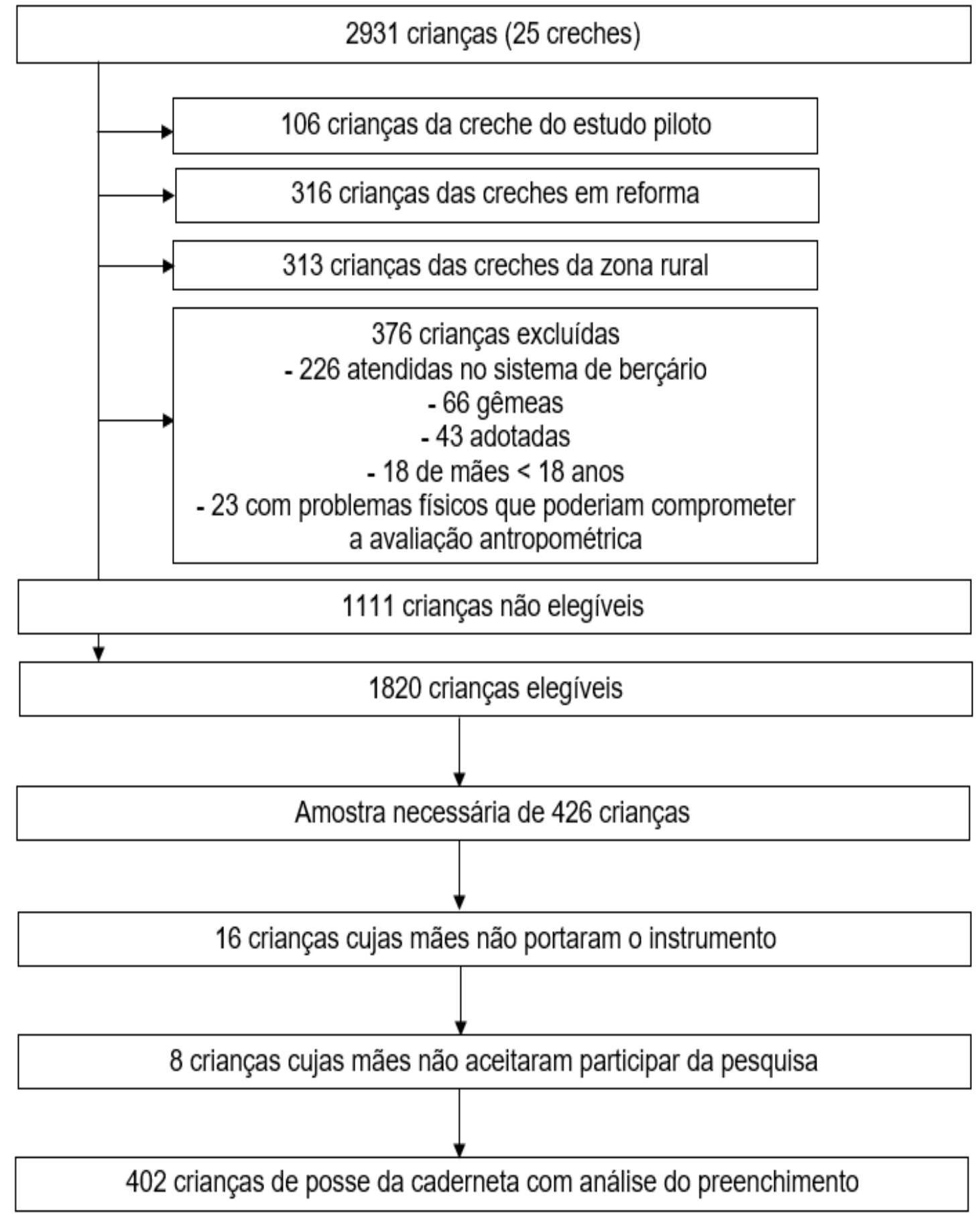

Fonte: Elaboração própria (2019). 
A informação sobre a data de nascimento das crianças foi obtida mediante verificação direta da CSC, ou da ficha de cadastro das creches para crianças sem posse do instrumento. A idade das crianças foi calculada pela diferença entre as datas de entrevista e de nascimento.

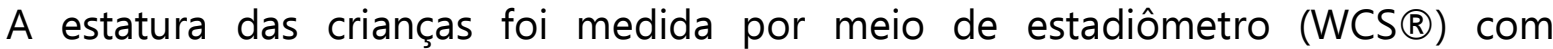
amplitude de $200 \mathrm{~cm}$ e subdivisões de $0,1 \mathrm{~cm}$. Todas as crianças foram pesadas utilizando balança eletrônica do tipo plataforma com capacidade para $150 \mathrm{~kg}$ e graduação em $100 \mathrm{~g}$ (Tanita UM-080®). Na pesagem foi permitida apenas uma peça íntima leve. As medições foram realizadas em duplicata por uma única dupla de profissionais de saúde treinados e padronizados por testes de exatidão e de precisão, de acordo com normas técnicas padronizadas. ${ }^{14}$ As médias das medidas de peso e de estatura foram as consideradas para o cálculo dos escores-Z de estatura/idade, peso/idade e Índice de Massa Corporal/idade (IMC/idade). Utilizou-se, para esses fins, o programa WHO Anthro Plus 2009. Tomou-se como referência a população do Multicentre Growth Reference Study (Who Child Growth Standards ${ }^{15}$ para crianças abaixo de cinco anos e Who Reference 5-19 years ${ }^{16}$ para crianças com cinco anos ou mais).

Foi verificado o preenchimento de 17 variáveis consideradas essenciais ao acompanhamento da saúde da criança e de preenchimento mínimo indispensável2: nome, data de nascimento, endereço, peso ao nascer, comprimento ao nascer, perímetro cefálico ao nascer, Apgar no $5^{\circ}$ minuto, tipo de parto, trimestre de início do pré-natal, número de consultas de pré-natal, idade gestacional, tipo de alimentação na alta da maternidade, registro do perímetro cefálico ao nascer no gráfico correspondente, última medida de perímetro cefálico marcada no gráfico correspondente (para crianças nos dois primeiros anos de vida), registro do peso ao nascer no gráfico correspondente, última medida de peso marcada no gráfico correspondente e desenvolvimento neuropsicomotor.

A avaliação do preenchimento dessas variáveis tomou como referência as orientações do Manual para Utilização da Caderneta de Saúde da Criança. ${ }^{17}$ Os itens foram 
considerados preenchidos corretamente quando estavam de acordo com as orientações contidas no manual. Quando não havia normalização sobre o assunto, consideraram-se critérios previamente estabelecidos pelo coordenador do projeto.

O nome e a data de nascimento da criança presentes na CSC foram comparados àqueles do Registro de Nascimento Civil, disponível na documentação exigida nas creches ao responsável pela criança no ato da matrícula. O nome foi considerado corretamente preenchido sempre que estava sem abreviatura, completo e sem permuta na sequência dos nomes e sobrenomes. A data de nascimento foi considerada de preenchimento correto quando possuía a sequência correta do dia/mês/ano.

O endereço foi avaliado quanto à existência do registro do nome da rua, número do domicílio e bairro, sem verificar a veracidade das informações. O preenchimento foi considerado correto quando fornecidas todas as informações.

Os registros de peso ao nascer (gramas), comprimento ao nascer (cm), perímetro cefálico ao nascer $(\mathrm{cm})$, Apgar no $5^{\circ}$ minuto, número de consultas de pré-natal e idade gestacional (semanas) foram considerados adequadamente preenchidos sempre que houvesse uma única anotação registrada em local apropriado. Para itens com unidade de referência, essas informações também deveriam estar corretas.

As variáveis tipo de parto, trimestre de início do pré-natal, tipo de alimentação na alta da maternidade e os registros do perímetro cefálico e peso ao nascer nos gráficos correspondentes foram consideradas apenas como preenchidas ou não preenchidas. Nestes casos, preenchimento e preenchimento correto foram adotados com o mesmo significado.

O preenchimento do peso no gráfico correspondente da CSC foi considerado correto quando existia no mínimo um registro na faixa etária da criança (2-3 anos, 3-4 anos, 4-5 anos). O último registro de perímetro cefálico no gráfico correspondente foi considerado corretamente preenchido quando existia alguma anotação na faixa etária de 18 a 24 meses. 
O desenvolvimento neuropsicomotor foi considerado corretamente preenchido sempre que existiam todos os registros sobre as habilidades esperadas em conformidade com a idade cronológica da criança, independente do marco ter sido presente, ausente ou não verificado. Como todas as crianças tinham no mínimo 24 meses de idade, considerouse preenchimento adequado quando todos os espaços disponíveis para anotações, até 2 a 3 anos ou 3 a 6 anos, dependendo da idade da criança, estavam preenchidos.

O escore de preenchimento da CSC foi calculado conforme proposta de Alves, et al. $^{2}$ Atribuiu-se valores um e zero para itens preenchidos corretamente e incorretamente, respectivamente. O somatório dos valores recebidos em cada item foi utilizado para indicar a pontuação final de preenchimento para cada CSC. Assim, admitiu-se variação de 0 a 17 pontos. Quanto maior o valor do escore, mais itens estavam preenchidos corretamente segundo os critérios adotados. Valores de escore superiores a oito pontos (47\% de itens preenchidos corretamente) foram definidos para denotar preenchimento adequado. A definição do ponto de corte foi feita com base na distribuição estatística dos valores de preenchimento, tendo em vista a inexistência de dados na literatura para esses fins.

Os dados foram digitados no programa Excel, com dupla digitação dos questionários. O aplicativo Validate do programa Epi-Info foi usado para verificar a consistência dos dados e gerar o banco final.

Para verificar diferenças nas médias dos escores-Z de estatura/idade, peso/idade e IMC/idade segundo o escore de preenchimento da CSC, utilizou-se o teste t de Student. Consideraram-se estatisticamente significantes valores de $p<5,0 \%$. As análises foram realizadas com a utilização do programa R-2.10.0.

A pesquisa foi aprovada pelo Comitê de Ética em Pesquisa da Universidade Estadual da Paraíba, protocolada com o número CAAE 0273.0.133.000-12. Todos os participantes assinaram o Termo de Consentimento Livre e Esclarecido. 


\section{Resultados}

Das 402 crianças, 174 (43,28\%) tinham de 36-72 meses e 210 (52,24\%) eram do sexo feminino. Todas as crianças eram acompanhadas pelas equipes de saúde da família. As médias \pm desvio-padrão (escore-Z) de estatura/idade, peso/idade e Índice de Massa Corporal/idade foram de $-0,49 \pm 1,09 ; 0,14 \pm 1,13$ e 0,22 $\pm 1,14$, respectivamente.

A situação de preenchimento da CSC é apresentada na Tabela 1. Maiores percentuais de preenchimento adequado encontraram-se nos aspectos do bloco de informações relacionadas à identificação da criança e às condições do recém-nascido. O peso ao nascer foi a de melhor preenchimento, em $97,8 \%$ das CSC, seguido da data de nascimento (96,8\%), comprimento ao nascer $(94,3 \%)$ e perímetro cefálico ao nascer $(87,8 \%)$. No bloco de informações sobre gravidez e parto, destacam-se baixos percentuais de preenchimento corretos para o trimestre de início de pré-natal (17,7\%) e o número de consultas de pré-natal (15,2\%), ambos relativos a informações da gravidez. Para as variáveis sobre o crescimento e desenvolvimento, o preenchimento adequado variou de $2 \%$ (idade em que a última medida de peso foi marcada no gráfico e anotações sobre o desenvolvimento neuropsicomotor) a $32,8 \%$ (registro do peso ao nascer no gráfico correspondente).

Tabela 1. Preenchimento correto dos itens da Caderneta de Saúde da Criança analisados. Campina Grande, Paraíba, Brasil, 2013

\section{Itens da Caderneta de Saúde da Criança}

ficação da criança e condições do recém-nascido

Nome

Data de nascimento

Endereço

Peso ao nascer

Comprimento ao nascer

Perímetro cefálico ao nascer

Apgar no $5^{\circ}$ minuto

Idade gestacional

Tipo de alimentação (alta da maternidade)

Gravidez e parto

Tipo de parto

Trimestre de início do pré-natal

\section{Preenchimento}

correto

n

$\%$

$319 \quad 79,4$

38896,6

$297 \quad 74$

$393 \quad 97,8$

$379 \quad 94,3$

$353 \quad 87,8$

$246 \quad 61,2$

$118 \quad 29,4$

$127 \quad 31,6$

$247 \quad 61,4$

$71 \quad 17,7$ 


\begin{tabular}{|c|c|c|}
\hline Número de consultas de pré-natal & 61 & 15,2 \\
\hline \multicolumn{3}{|l|}{ Crescimento e desenvolvimento da criança } \\
\hline Registro do perímetro cefálico ao nascer no gráfico & 55 & 13,7 \\
\hline $\begin{array}{l}\text { Última medida de perímetro cefálico marcada no } \\
\text { gráfico (para crianças nos dois primeiros anos de vida) }\end{array}$ & 32 & 8 \\
\hline Registro do peso ao nascer no gráfico & 132 & 32,8 \\
\hline Última medida de peso marcada no gráfico & 8 & 2 \\
\hline Desenvolvimento neuropsicomotor & 8 & 2 \\
\hline
\end{tabular}

Fonte: Elaboração própria (2019).

Crianças com escore de preenchimento da CSC adequado apresentaram média de escores-Z de estatura/idade significativamente maior do que crianças classificadas com preenchimento inadequado (Tabela 2).

Tabela 2. Médias e desvios-padrão dos escores-Z para os índices estatura para idade (E/I), peso para idade (P/I) e Índice de Massa Corporal para idade (IMC/I) de crianças segundo o escore de preenchimento da Caderneta de Saúde da Criança (CSC). Campina Grande, Paraíba, Brasil, 2013

\begin{tabular}{|c|c|c|c|c|}
\hline \multirow{2}{*}{ Escore de preenchimento* } & \multirow{2}{*}{$\begin{array}{c}\text { CsC } \\
n\end{array}$} & \multicolumn{3}{|c|}{ Média \pm Desvio-padrão (escore-Z) } \\
\hline & & $E / I$ & $\mathbf{P} / \mathbf{I}$ & IMC/I \\
\hline Inadequado & 252 & $-0,69 \pm 1,22$ & $0,06 \pm 1,62$ & $0,27 \pm 1,07$ \\
\hline Adequado & 150 & $-0,37 \pm 1,02$ & $0,09 \pm 1,32$ & $0,30 \pm 1,14$ \\
\hline $\mathrm{p}$-valor** & - & 0,031 & 0,701 & 0,761 \\
\hline
\end{tabular}

*Sem diferenças segundo sexo e faixa etária. **Teste t de Student. Fonte: Elaboração própria (2019).

\section{Discussão}

Analisando-se os índices antropométricos das crianças do atual estudo observam-se médias que correspondem ao esperado para a idade (assumindo-se \pm 2 escore- $Z$ como ponto de corte). A menor média que correspondeu ao escore-Z de estatura/idade $(-0,49)$ está acima da média nacional $(-1,32){ }^{18}$

Ao considerar os percentuais de preenchimento das variáveis de análise da CSC encontrados neste estudo, o nome e a data de nascimento da criança foram preenchidos adequadamente em proporções inferiores às apresentadas por outros pesquisadores. ${ }^{2,19}$ É possível que os menores percentuais aqui reportados estejam relacionados às 10 hittps://doio.org/10.14295//mphc.v10io.0.957 
especificidades exigidas para se considerar o preenchimento correto, principalmente em relação ao nome da criança, o que é preocupante dada a relevância dessa informação para o contato com a família e devolução do instrumento em casos de extravio. ${ }^{4}$ No entanto, a informação sobre o endereço da criança apresentou melhor situação que em outras localidades. $^{4,19}$ Assim, oportuniza-se a continuidade da assistência ao se facilitar a comunicação do sistema de saúde com a criança e servir de informação sobre o nível de risco socioambiental. ${ }^{4}$

Neste estudo, o preenchimento das informações sobre início e número de consultas de pré-natal pode ser considerado insatisfatório se comparados com proporções de adequação superiores a 50\% reportadas em outras pesquisas. ${ }^{2,4}$ Para a variável tipo de parto, resultados de trabalhos anteriores assemelham-se aos atuais, destacando-se melhor preenchimento, mas ainda distante da obrigatoriedade estabelecida. ${ }^{2,4,20}$ Negligências nos registros dessas informações são preocupantes, devido à relevância das condições da gravidez e do parto na prevenção de morbidades e morte perinatal. ${ }^{3}$

Apesar da importância do registro da alimentação da criança na alta da maternidade como meio de permitir a intervenção imediata da equipe de saúde diante das questões relativas à amamentação, ${ }^{4} 68 \%$ das CSC observadas não tinham as informações. Esse sub-registro vem sendo confirmado na literatura. ${ }^{2,4}$

Com relação ao preenchimento das condições de nascimento, os melhores percentuais para o peso e o comprimento coincidem com os resultados de estudos anteriores. $^{2,4}$ Essa realidade pode estar relacionada à maior incorporação desses registros na rotina dos profissionais de saúde como consequência da inclusão do peso e comprimento ao nascer desde o surgimento do CC em decorrência da concepção da vigilância do crescimento como ação pioneira e básica de monitoramento da saúde da criança. ${ }^{1}$

A idade gestacional apresentou proporção de preenchimento adequado bem inferior ao encontrado em outras investigações de $70 \%$ ou mais. ${ }^{2,4} \mathrm{~A}$ melhoria do 
preenchimento desta variável é fundamental, pois a mesma é indispensável para o conhecimento relacionado ao nascimento pré-termo que constitui um problema de saúde pública que aumenta mundialmente, inclusive no Brasil. A prematuridade representa uma das principais causas de mortalidade infantil e um importante fator de risco para morbidade neonatal e infantil. ${ }^{21}$

Neste estudo, o índice de Apgar no $5^{\circ}$ minuto foi preenchido em $61,2 \%$ dos casos, valor superior ao encontrado em Belo Horizonte (MG) $(53,5 \%),{ }^{2}$ porém inferior a outro estudo na mesma cidade $(74,0 \%) .{ }^{4}$ A inexistência dessa informação pode representar a possibilidade das crianças não serem assistidas adequadamente ao nascer, prejudicando a identificação de condições adversas ocorridas ao nascimento, o acompanhamento da saúde da criança e a adoção de medidas que devem ser tomadas precocemente como a reanimação. ${ }^{22}$ Cabe destacar que a falta desta informação não implica necessariamente que o índice não foi avaliado, sendo normatizado pelo Ministério da Saúde que todo parto tem que ter a assistência do pediatra. ${ }^{23}$

O baixo percentual de preenchimento correto das informações sobre o desenvolvimento neuropsicomotor registrado converge com investigações realizadas em outras cidades brasileiras. ${ }^{2,5,6,24}$ Essa condição prejudica a identificação precoce de distúrbios do desenvolvimento e, portanto, a adoção de intervenções de baixo custo e potencial de impacto nos casos necessários. ${ }^{6}$ Talvez, o desconhecimento dos profissionais de saúde sobre desenvolvimento infantil e suas formas de avaliação possa contribuir negativamente na sua valorização ${ }^{3}$, mas isso ficou fora do alcance deste estudo.

Considerando os parâmetros relacionados ao crescimento, ressalta-se nos resultados do atual estudo a subutilização dos gráficos da CSC, coincidindo com relatos anteriores. ${ }^{2,6,19,20,24-27}$ O preenchimento inadequado das curvas de crescimento também foi debatido em artigo de revisão específico sobre o tema ${ }^{8}$ e destacado como um dos principais problemas na vigilância do crescimento da rede básica de saúde. ${ }^{9}$ Essa situação é preocupante, dada a importância do monitoramento do crescimento na detecção 
oportuna de problemas de crescimento e desenvolvimento, para a saúde da criança e no estabelecimento de vínculos com a família. ${ }^{7,9,20}$

Cabe ressaltar que no atual estudo o peso e o perímetro cefálico ao nascer, registrados corretamente em $97,8 \%$ e $87,8 \%$ dos casos, respectivamente, nas informações do recém-nascido, apenas 32,8\% e 13,7\% dos registros estavam devidamente contidos nos seus respectivos gráficos, o que reforça as deficiências na utilização dos mesmos. Resultado semelhante foi observados por outros pesquisadores, ${ }^{2}$ o que conforma um quadro preocupante devido à relação do peso e perímetro cefálico ao nascer com o desenvolvimento da criança e o risco de déficits de crescimento e de mortalidade infantil como consequência de nascimentos com pesos inferiores aos considerados adequados para a saúde. ${ }^{9}$ Além disso, o perímetro cefálico representa um importante indicador de desenvolvimento mental. ${ }^{28}$

Até onde é de conhecimento dos autores, este é primeiro estudo que analisa a associação entre índices antropométricos de crianças brasileiras e o preenchimento da CSC. Considerando que a estatura/idade da criança apresentou melhor situação entre aquelas em que o preenchimento da CSC foi adequado, é possível postular que o crescimento da criança seja uma medida sensível aos cuidados de saúde. A atribuição do crescimento como indicador da situação de saúde e a importância das questões de alimentação e nutrição na CSC podem justificar esses achados., ${ }^{4,7,19}$ Além disso, experiências de outros países tem indicado que práticas adequadas de vigilância do crescimento podem favorecer o estado nutricional das crianças. ${ }^{10}$

Deve-se ter presente que o ponto de corte usado para indicar adequação do preenchimento da CSC neste estudo pode ser considerado baixo, o que questiona a possibilidade de assumir os problemas de preenchimento como sinal de pior assistência. Somam-se aos anteriores argumentos as limitações condicionadas a não realização de análises sobre as influências de fatores socioeconômicos e da qualidade das creches que também podem determinar alterações do crescimento da criança. Além disso, há que 
destacar que as médias de escores-Z de estatura/idade dos dois grupos estiveram dentro dos limites da normalidade, não sendo a diferença de relevância clínica.

A interpretação dos resultados deste estudo deve considerar que a utilização adequada da CSC compete a todos os profissionais que assistem a criança e à família. Nesse sentido, o registro das informações é essencial para a comunicação entre os profissionais e o compartilhamento de informações em saúde com a família e cuidadores (creches) direcionados ao acompanhamento integral da saúde infantil. 2,17,19,29

Neste trabalho devem ser consideradas as limitações impostas pelas restrições na capacidade de análise causal próprias do delineamento. Além disso, fatores como o papel do cuidador da criança e características dos serviços de saúde, inclusive dos profissionais, não foram contemplados. Há necessidade de considerar, ainda, a possibilidade de distorções devidas ao uso de dados secundários que podem ter sua qualidade comprometida.

\section{Conclusão}

Os achados apresentados evidenciam entre crianças assistidas em creches deficiências de preenchimento da CSC que compreendem parâmetros do crescimento e desenvolvimento. Essas deficiências podem estar associadas ao crescimento das crianças, hipótese que precisa ser testada por meio de pesquisas com foco em modelos de intervenção e com o devido controle de fatores de confusão.

\section{Referências}

1. Andrade GN, Rezende TMRL, Madeira AMF. Caderneta de saúde da criança: experiências dos profissionais da atenção primária à saúde. Rev Esc Enferm USP. 2014;48(5):857-64. http://dx.doi.org/10.1590/S0080-6234201400005000012.

2. Alves CRL, Lasmar LMLBF, Goulart LMHF, Alvim CG, Maciel GVR, Viana MRA, et al. Qualidade do preenchimento da Caderneta de Saúde da Criança e fatores associados. Cad Saude Publica. 2009;25(3):583-95. http://dx.doi.org/10.1590/S0102- 
$311 \times 2009000300013$.

3. Brasil, Ministério da Saúde, Secretaria de Atenção à Saúde, Departamento de Atenção Básica. Saúde da criança: crescimento e desenvolvimento. Brasília, DF: Ministério da Saúde; 2012. (Cadernos de Atenção Básica; vol. 33).

4. Goulart LMHF, Alves CRL, Viana MRA, Moulin, ZS, Carmo GAA, Costa JG, et al. Caderneta de Saúde da Criança: avaliação do preenchimento dos dados sobre gravidez, parto e recém-nascido. Rev Paul Pediatr. 2008;26(2):106-12. http://dx.doi.org/10.1590/S010305822008000200002.

5. Costa JSD, Cesar JÁ, Pattussi MP, Fontoura LP, Barazzetti L, Nunes MF, et al. Assistência à criança: preenchimento da caderneta de saúde em municípios do semiárido brasileiro. Rev Bras Saude Matern Infant. 2014;14(3):219-27. http://dx.doi.org/10.1590/S151938292014000300003.

6. Ceia MLM, Cesar JA. Avaliação do preenchimento dos registros de puericultura em unidades básicas de saúde em Pelotas, RS. Rev AMRIGS. 2011;55(3):244-9.

7. Rocha $A C D$, Figueroa Pedraza D. Acompanhamento do crescimento infantil em unidades básicas de saúde da família do município de Queimadas, Paraíba, Brasil. Texto Cont Enferm. 2013;22(4):1169-78. http://dx.doi.org/10.1590/S0104-07072013000400036.

8. Almeida AC, Mendes LC, Sad IR, Ramos EG, Fonseca VM, Peixoto MVM. Uso de instrumento de acompanhamento do crescimento e desenvolvimento da criança no Brasil - Revisão sistemática de literatura. Rev Paul Pediatr. 2016;34(1):122-31. http://dx.doi.org/10.1016/j.rppede.2015.12.002.

9. Figueroa Pedraza D. Growth surveillance in the context of the Primary Public Healthcare Service Network in Brazil: literature review. Rev Bras Saude Matern Infant. 2016;16(1):719. http://dx.doi.org/10.1590/1806-93042016000100002.

10. Mangasaryan N, Arabi M, Schultink W. Revisiting the concept of growth monitoring and its possible role in community-based nutrition programs. Food Nutr Bull. 2011;32(1):4253. http://dx.doi.org/10.1177/156482651103200105.

11. Figueroa Pedraza D, Souza MM, Rocha ACD. Fatores associados ao estado nutricional de crianças pré-escolares brasileiras assistidas em creches públicas: uma revisão sistemática. Rev Nutr. 2015;28(4):451-64. http://dx.doi.org/10.1590/141552732015000400010 .

12. Maranhão DG, Sarti CA. Creche e família: uma parceria necessária. Cad Pesqui.

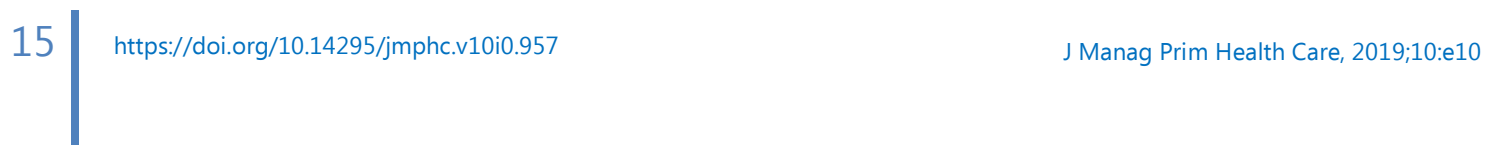


2008;38(133):171-94. http://dx.doi.org/10.1590/S0100-15742008000100008.

13. Leite RFB, Veloso TMG. Limites e avanços do Programa Saúde da Família de Campina Grande: um estudo a partir de representações sociais. Saude Soc. 2009;18(1):50-62. http://dx.doi.org/10.1590/S0104-12902009000100006.

14. Brasil, Ministério da Saúde, Secretaria de Atenção à Saúde, Departamento de Atenção Básica. Orientações para a coleta e análise de dados antropométricos em serviços de saúde: Norma Técnica do Sistema de Vigilância Alimentar e Nutricional - SISVAN. Brasília, DF: Ministério da Saúde; 2011.

15. World Health Organization. WHO Child Growth Standards. Length/height-for-age, weight-for-age, weight-for-length, weight-for-height and body mass index-for-age. Methods and development. Geneva: WHO; 2006.

16. Onis M, Onyango AW, Borghi E, Siyam A, Nishida C, Siekmanna J. Development of a WHO growth reference for school-aged children and adolescents. Bull World Health Org. 2007;85(9):660-7. http://dx.doi.org/10.2471/blt.07.043497.

17. Brasil, Ministério da Saúde, Secretaria de Atenção à Saúde, Departamento de Ações Programáticas Estratégicas, Manual para utilização da Caderneta de Saúde da Criança. Brasília, DF: Ministério da Saúde; 2005.

18. Instituto Brasileiro de Geografia e Estatística. Antropometria e análise do estado nutricional de crianças e adolescentes no Brasil. Brasília, DF: IBGE; 2006.

19. Linhares AO, Gigante DP, Bender E, Cesar JÁ. Avaliação dos registros e opinião das mães sobre a caderneta de saúde da criança em unidades básicas de saúde, Pelotas, RS. Rev AMRIGS. 2012;56(3):245-50.

20. Faria M, Nogueira TA. Avaliação do uso da caderneta de saúde da criança nas unidades básicas de saúde em um munícipio de Minas Gerais. Rev Bras Cien Saude. 2013;11(38):815. https://doi.org/10.13037/rbcs.vol11n38.1944.

21. Assunção PL, Novaes HMD, Alencar GP, Melo ASO, Almeida MF. Desafios na definição da idade gestacional em estudos populacionais sobre parto pré-termo: o caso de um estudo em Campina Grande (PB), Brasil. Rev Bras Epidemiol. 2011;14(3):455-66. http://dx.doi.org/10.1590/S1415-790X2011000300010.

22. Oliveira TG, Freire PV, Moreira FT, Moraes JSB, Arrelaro RC, Rossi S, et al. Escore de Apgar e mortalidade neonatal em um hospital localizado na zona sul do município de São Paulo. Einstein. 2012;10(1):22-8. http://dx.doi.org/10.1590/S1679- 
45082012000100006

23. Brasil, Ministério da Saúde. Portaria n 569 de 01 de junho de 2000. Instituição do Programa de Humanização do Pré-natal e Nascimento no âmbito do Sistema Único de Saúde. Diário Oficial da União. Brasília, DF (8 jun. 2000); Sec. 1:4.

24. Abud SM, Gaíva MAM. Registro dos dados de crescimento e desenvolvimento na caderneta de saúde da criança. Rev Gaucha Enferm. 2015;36(2):97-105. http://dx.doi.org/10.1590/1983-1447.2015.02.48427.

25. Amorim LP, Senna MIB, Soares ARS, Carneiro GTN, Ferreira EF, Vasconcelos M, et al. Avaliação do preenchimento da caderneta de saúde da criança e qualidade do preenchimento segundo o tipo de serviço de saúde usado pela criança. Cienc Saude Colet. 2018;23(2):585-97. http://dx.doi.org/10.1590/1413-81232018232.06962016.

26. Palombo CNT, Duarte LS, Fujimori E, Toriyama ATM. Uso e preenchimento da Caderneta de Saúde da Criança com foco no crescimento e desenvolvimento. Rev Esc Enferm USP. 2014;48(Esp):60-7. http://dx.doi.org/10.1590/S0080-623420140000600009.

27. Araujo EMN, Gouveia MTO, Figueroa Pedraza D. Use of a child health surveillance instrument focusing on growth. A cross-sectional study. Sao Paulo Med J. 2017;135(6):541-7. http://dx.doi.org/10.1590/1516-3180.2016.0345120617.

28. Governo do Estado do Rio Grande do Norte, Secretaria de Estado da Saúde Pública. Protocolo para investigação de casos de microcefalia. [Natal]: Secretaria de Estado da Saúde Pública; 2015.

29. Silva FB, Gaíva AMM, Mello DF. Utilização da caderneta de saúde da criança pela família: Percepção dos profissionais. Texto Contexto Enferm. 2015;24(2):407-14. http://dx.doi.org/10.1590/0104-07072015000212014. 


\section{Minicurrículo}

Dixis Figueroa Pedrazza | ORCiD: 0000-0002-5394-828X

Doutor em Nutrição pela Universidade Federal de Pernambuco.

Professor do Departamento de Enfermagem da Universidade Estadual da Paraíba.

Professor do Programa de Pós-graduação em Saúde da Família no Nordeste - Rede Nordeste de Formação em Saúde da Família. 